\title{
Stereotactic radiotherapy of intrapulmonary lesions: comparison of different dose calculation algorithms for Oncentra MasterPlan ${ }^{\circledast}$
}

Almut Troeller*, Sylvia Garny, Sophia Pachmann, Steffi Kantz, Sabine Gerum, Farkhad Manapov, Ute Ganswindt, Claus Belka and Matthias Söhn

\begin{abstract}
Background: The use of high accuracy dose calculation algorithms, such as Monte Carlo (MC) and Collapsed Cone (CC) determine dose in inhomogeneous tissue more accurately than pencil beam (PB) algorithms. However, prescription protocols based on clinical experience with PB are often used for treatment plans calculated with CC. This may lead to treatment plans with changes in field size (FS) and changes in dose to organs at risk (OAR), especially for small tumor volumes in lung tissue treated with SABR.

Methods: We re-evaluated 17 3D-conformal treatment plans for small intrapulmonary lesions with a prescription of $60 \mathrm{~Gy}$ in fractions of $7.5 \mathrm{~Gy}$ to the $80 \%$ isodose. All treatment plans were initially calculated in Oncentra MasterPlan ${ }^{\oplus}$ using a PB algorithm and recalculated with $\mathrm{CC}\left(\mathrm{CC}_{\text {re-calc }}\right)$. Furthermore, a CC-based plan with coverage similar to the PB plan $\left(\mathrm{CC}_{\text {cov }}\right)$ and a $\mathrm{CC}$ plan with relaxed coverage criteria $\left(\mathrm{CC}_{\mathrm{clin}}\right)$, were created. The plans were analyzed in terms of $D_{\text {mean }}, D_{\text {min }}, D_{\text {max }}$ and coverage for GTV, PTV and ITV. Changes in mean lung dose (MLD), $V_{10 G y}$ and $V_{20 G y}$ were evaluated for the lungs. The re-planned CC plans were compared to the original PB plans regarding changes in total monitor units (MU) and average FS.

Results: When PB plans were recalculated with CC, the average V $_{60 G y}$ of GTV, ITV and PTV decreased by 13.2\%, $19.9 \%$ and $41.4 \%$, respectively. Average $D_{\text {mean }}$ decreased by $9 \%$ (GTV), 11.6\% (ITV) and 14.2\% (PTV). $D_{\min }$ decreased by $18.5 \%$ (GTV), $21.3 \%$ (ITV) and 17.5\% (PTV). $D_{\max }$ declined by 7.5\%. PTV coverage correlated with PTV volume $(p<0.001)$. MLD, $V_{10 G y}$ and $V_{20 G y}$ were significantly reduced in the $C C$ plans. Both, $\mathrm{CC}_{\text {cov }}$ and $\mathrm{CC}_{\text {clin }}$ had significantly increased MUs and FS compared to PB.
\end{abstract}

Conclusions: Recalculation of PB plans for small lung lesions with CC showed a strong decline in dose and coverage in GTV, ITV and PTV, and declined dose in the lung. Thus, switching from a PB algorithm to CC, while aiming to obtain similar target coverage, can be associated with application of more $\mathrm{MU}$ and extension of radiotherapy fields, causing greater OAR exposition.

Keywords: Lung SBRT, Dose calculation algorithms, Collapsed Cone, Pencil Beam, Small pulmonary lesions

*Correspondence: almut.troeller@med.uni-muenchen.de

Department of Radiation Oncology, University of Munich, Marchioninistr. 15,

81377 Munich, Germany 


\section{Background}

The use of high accuracy dose calculation algorithms, such as Monte Carlo (MC) and Collapsed Cone (CC) has increased during the past few years due to increased speed of the algorithms and availability of advanced computational technology. These algorithms are capable of estimating dose in inhomogeneous media more accurately than simpler dose calculation algorithms of pencil beam $(\mathrm{PB})$ type. It is well known that the pencil beam algorithms lack accurate modeling of lateral scatter and -backscatter, because they neglect tissue density inhomogeneity in directions other than the beam and assume water density instead. In contrast, CC and MC include more accurate inhomogeneity corrections [1]. MC simulations, which explicitly model particle interactions and transport in the patient, are generally considered the gold standard of dose calculation. A popular choice for dose calculation are $\mathrm{CC}$ based algorithms that approximately model lateral scattering and -backscatter via density dependent anisotropic rescaling of pre-calculated point kernels for effects from secondary particle transport. In the following, we investigate dose calculation differences between $\mathrm{PB}$ and $\mathrm{CC}$, which are the two dose calculation algorithms choices implemented in our clinical 3D-CRT treatment planning system (TPS) Oncentra MasterPlan ${ }^{\circ}$ (OTP, Version 4.2, Nucletron).

Differences in dose calculation accuracy in the patient between the different algorithms can be expected to be most predominant in anatomic regions with large local tissue density inhomogeneities and density discontinuities, such as small lung lesions surrounded by low density lung tissue. When stereotactic radiotherapy of intrapulmonary lesions was first introduced into routine clinical treatment, most institutions calculated dose distributions using dose calculation programs based on PB type algorithms. However, it is now widely and clinically accepted that dose calculation algorithms of the $\mathrm{PB}$ type generally do not provide enough accuracy for dose calculation in inhomogeneous tissue [2]. Recent recommendations for the implementation of stereotactic ablative radiotherapy (SABR) of lung cancer therefore include the use of those adapted algorithms. However, historically, prescription schemes were based on clinical experience with $\mathrm{PB}$. Recent guidelines such as the ICRU (ICRU-62, Bethesda, Md, USA, 1999) recommend similarly strict requirements for treatment plans calculated with CC or MC. Because of the insufficient modeling of lateral scattering and -backscatter, the dose to the tumor is usually overestimated when using $\mathrm{PB}$ and the same plan may indicate lower dose when recalculated with $\mathrm{CC}[3,4]$. This will lead to treatment plans with changes in field size and MUs delivered, as well as changes in dose to organs at risk (OAR) when creating a treatment plan based on a CC dose calculation algorithm without proper adjustment of the prescription.
In order to quantify differences between dose distributions calculated with $\mathrm{PB}$ and $\mathrm{CC}$ in patients treated at our institution, we examined treatment plans of patients that received SABR of intrapulmonary lesions. These patients usually have smaller tumors and may show large discrepancies between dose calculated with $\mathrm{PB}$ and $\mathrm{CC}$. There is a variety of different implementations of PB algorithms, that may differ in how severely calculated dose differs from delivered dose as estimated with $\mathrm{CC}$. In this study we investigate the enhanced $\mathrm{PB}$ and $\mathrm{CC}$ algorithms used in the Oncentra MasterPlan ${ }^{\circ}$ treatment planning system.

\section{Methods}

The treatment plans of 17 patients treated for small intrapulmonary lesions at our institution between 2008 and 2010 were retrospectively evaluated (Table 1). For each patient 3 computed tomography (CT) scans with a $3 \mathrm{~mm}$ slice gap were acquired prior to treatment: a freebreathing scan, a maximum inhale and a maximum exhale scan. The scans were then imported to the Oncentra MasterPlan ${ }^{\circ}$ treatment planning system (Version 4.2, Nucletron). The gross tumor volume (GTV) was contoured on each of the CT scans and an internal target volume (ITV) was defined by forming the union of all GTVs. The planning target volume (PTV) was created from the ITV, by adding a $7 \mathrm{~mm}$ margin laterally and $9 \mathrm{~mm}$ in the cranial-caudal direction. For all patients, a

Table 1 Tumor size, volume and location for all patients

\begin{tabular}{lllll}
\hline Patient & $\begin{array}{l}\text { Maximum tumor } \\
\text { diameter }(\mathbf{c m})\end{array}$ & $\begin{array}{l}\text { Total volume } \\
\text { GTV }(\mathbf{c c m})\end{array}$ & $\begin{array}{l}\text { Tumor } \\
\text { location }\end{array}$ & $\begin{array}{l}\text { Central/ } \\
\text { peripheral }\end{array}$ \\
\hline 1 & 1.6 & 2.0 & RLL & peripheral \\
2 & 1.6 & 3.5 & RUL & central \\
3 & 4.1 & 41.2 & RUL & peripheral \\
4 & 3.1 & 12.1 & LLL & peripheral \\
5 & 2.8 & 6.4 & $\mathrm{R}$ & central \\
6 & 1.5 & 1.4 & RUL & peripheral \\
7 & 1.2 & 4.6 & RLL & peripheral \\
8 & 2.5 & 3.8 & $\mathrm{R}$ & central \\
9 & 1.2 & 1.2 & $\mathrm{R}$ & central \\
10 & 2.6 & 15.2 & $\mathrm{RLL}$ & peripheral \\
11 & 3.0 & 17.0 & $\mathrm{LUL}$ & central \\
12 & 2.5 & 4.4 & $\mathrm{RUL}$ & peripheral \\
13 & 2.5 & 27.0 & $\mathrm{RLL}$ & peripheral \\
14 & 3.9 & 27.9 & $\mathrm{RLL}$ & peripheral \\
15 & 4.6 & 61.6 & $\mathrm{RLL}$ & central \\
16 & 1.8 & 4.7 & $\mathrm{RUL}$ & peripheral \\
17 & 4.0 & 36.2 & $\mathrm{LUL}$ & peripheral \\
\hline
\end{tabular}

${ }^{\dagger}$ For the purpose of this study we defined tumors that were directly attached to the mediastinum or pleura as peripheral and all others (fully surrounded by low density lung tissue) as central. 
cumulative physical dose of up to 60 Gy was prescribed to the planning target volume (PTV) in single fractions of $7.5 \mathrm{~Gy}$, such that the $80 \%$ isodose line completely covered the PTV.

All dose calculations were performed on the freebreathing $\mathrm{CT}$ scan without density overrides. The 3D conformal, un-modulated treatment plans were designed for a Siemens Oncor treatment unit with a $10 \mathrm{~mm}$ leafwidth multi-leaf collimator (MLC). Mixed photon energy was used (6 and $15 \mathrm{MV}$ ) incorporating 7 beams on average. The beam model was verified against experiment and accepted for clinical use.

The dose distributions of the original treatment plans were calculated using OTP's enhanced pencil beam (PB) algorithm. For the purpose of this study, all plans were

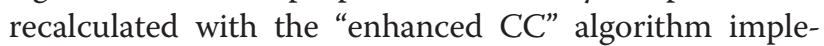
mented in OTP $\left(\mathrm{CC}_{\text {re-calc }}\right)$, without altering the field shape, field size, beam setup, or monitor units. Furthermore we created two additional plans calculated with CC for each patient: A plan that resembles what would currently be considered acceptable for a patient in our clinic $\left(\mathrm{CC}_{\mathrm{clin}}\right)$ and a stricter second plan $\left(\mathrm{CC}_{\mathrm{cov}}\right)$ that obtained coverage similar to the original $\mathrm{PB}$ plan. The former constitutes a clinically accepted compromise in our institution that may formally violate the PTV coverage criteria mentioned above in low density parts of the PTV where full dose build-up is difficult to realize for physical reasons. The latter was created to demonstrate how dose to organs at risk would change in the hypothetical scenario of switching from a PB algorithm to a $\mathrm{CC}$ algorithm without adapting the criteria for an acceptable plan.

For the $\mathrm{CC}_{\text {clin }}$ and $\mathrm{CC}_{\text {cov }}$ plans MUs as well as field size and shape had to be adjusted accordingly. For the $\mathrm{CC}_{\text {cov }}$ the fields were opened in beam's eye view (BEV) until coverage was achieved, while for the $\mathrm{CC}_{\text {clin }}$ the distance between the jaws and the PTV-outline were not to exceed $1 \mathrm{~cm}$ in BEV. All four plans per patient were then compared to each other. Parameters used for comparison were volume coverage $\left(\mathrm{V}_{60 \mathrm{~Gy}}\right)$, mean dose $\left(\mathrm{D}_{\text {mean }}\right)$, dose received by $99 \%$ and $1 \%$ of the volume $\left(D_{99}\right.$ and $\left.D_{1}\right)$, minimum dose $\left(D_{\min }\right)$, and maximum dose $\left(D_{\max }\right)$ for the GTV, ITV and PTV. Estimated coverage in dependence on GTV volume was also considered. Furthermore we evaluated $D_{\text {mean }}, V_{20 \text { Gy }}$ and $V_{10 \text { Gy }}$ to the lungs, $D_{\text {mean }}$ to the heart and $D_{\max }$ to the spinal cord and esophagus. Both lungs were contoured as one organ including the PTV. The adjusted $\mathrm{CC}_{\mathrm{clin}}$ and $\mathrm{CC}_{\mathrm{cov}}$ treatment plans were further analyzed regarding alterations of $\mathrm{MU}$ and field size compared to the original PB plan. The influence of tumor position and movement on coverage was briefly investigated. For the purpose of this study we defined tumors that were directly attached to the mediastinum or pleura as peripheral and all others as central. Statistical significance of the differences between parameters calculated with $\mathrm{PB}$ and parameters calculated with $\mathrm{CC}_{\text {re-calc }}$, $\mathrm{CC}_{\text {clin }}$ and $\mathrm{CC}_{\text {cov }}$ was determined using the Wilcoxon signed-rank test $(\mathrm{p}<0.05$ signifies significant difference). Correlation of the difference in target coverage between $\mathrm{PB}$ and $\mathrm{CC}$ (i.e. $\mathrm{V}_{60} \mathrm{~Gy}, \mathrm{~PB}-\mathrm{V}_{60 \mathrm{~Gy}, \mathrm{CC}}$ ) with the target ROI volumes was determined using Spearman's rank correlation coefficient, $\rho$. The significance of the correlation was verified with Spearman's rank test, with the null hypothesis $\mathrm{H}_{0}$, that $\mathrm{ROI}$ volume and difference in coverage are independent and alternative hypothesis $\mathrm{H}_{\mathrm{a}}$, that they are dependent. Analysis of the parameters and statistical analyses were performed in Mathematica (Wolfram Research, Inc., Mathematica, Version 9.0.1, Champaign, IL (2013)).

\section{Results and discussion}

Original PB plan vs. $\mathrm{CC}_{\text {re-calc }}$

For the target structures GTV, ITV and PTV all evaluated parameters $\left(\mathrm{V}_{60 \mathrm{~Gy}}, \mathrm{D}_{\text {mean }}, \mathrm{D}_{\min }, \mathrm{D}_{\max }, \mathrm{D}_{99}, \mathrm{D}_{1}\right)$ were significantly lower for the plans that were re-calculated based on the $\mathrm{CC}$ algorithm $\left(\mathrm{CC}_{\text {re-calc }}\right)$ as opposed to the original ones incorporating the $\mathrm{PB}$ algorithm $(\mathrm{p}<0.001)$.

All OAR parameters considered in this study $(\mathrm{p}<0.01)$, except $D_{\max }$ to the spinal cord, were statistically significantly lower when the PB plan was recalculated with CC. However, the differences regarding heart and spinal cord were of relatively small magnitude and may not be clinically relevant.

The results for all structures are shown in Table 2.

The GTV, ITV and PTV coverage with 60 Gy and higher as well as $\mathrm{D}_{99}$ and $\mathrm{D}_{\max }$ versus the GTV, ITV and PTV volume for both $\mathrm{PB}$ and $\mathrm{CC}_{\text {re-calc }}$ treatment plans are shown in Figures 1 and 2. For the PTV, a larger discrepancy between $\mathrm{PB}$ and $\mathrm{CC}$ coverage is significantly correlated with a smaller ROI volume $\left(\rho_{\mathrm{PTV}}=-0.77\right.$, $\mathrm{P}_{\text {PTV }}<0.001$ ), while for the GTV and ITV the correlation coefficient is less pronounced and not significant $\left(\rho_{\mathrm{ITV}}=-0.43, \quad \mathrm{p}_{\mathrm{PTV}}=0.08, \quad \rho_{\mathrm{GTV}}=-0.18, \quad \mathrm{p}_{\mathrm{GTV}}=0.49\right)$. Large difference of $\mathrm{D}_{\min }, \mathrm{D}_{99}, \mathrm{D}_{\max }$ and $\mathrm{D}_{1}$ between $\mathrm{PB}$ and CC is significantly correlated with ROI volume for all three target ROIs: GTV, ITV and PTV.

There was considerable variability of the dose volume parameters of GTV, ITV and PTV between patients, likely due to tumor volume and position. As expected the differences were more pronounced for tumors completely surrounded by lung tissue and not adherent to pleural or mediastinum tissues. For example, the difference in average GTV relative volume covered by 60 Gy or more was $3.5 \%$ for peripheral tumors compared to $6.1 \%$ for centrally located tumors. Presumably, this is due to the fact that the density of the chest wall and mediastinal tissue is closer to the density of water, than the low density lung tissue. Therefore the PB algorithm, which assumes water density laterally, estimates dose 
Table 2 Dose and volume parameters for targets and organs at risk for pencil beam and cc calculations

\begin{tabular}{|c|c|c|c|c|c|}
\hline ROI & Parameter & PB & $\mathrm{CC}_{\text {re-calc }}$ & $\mathrm{CC}_{\text {cov }}$ & $\mathrm{CC}_{\mathrm{clin}}$ \\
\hline \multirow[t]{6}{*}{ GTV } & $V_{60 G y}(\%)$ & $99.8 \pm 0.9$ & $86.6 \pm 26.5$ & $99.6 \pm 1.0$ & $99.4 \pm 1.5$ \\
\hline & $D_{\text {mean }}(G y)$ & $73.7 \pm 1.6$ & $67.0 \pm 4.4$ & $72.8 \pm 1.5$ & $72.5 \pm 1.6$ \\
\hline & $D_{\min }(G y)$ & $63.1 \pm 11.0$ & $51.4 \pm 9.6$ & $61.3 \pm 8.5$ & $59.5 \pm 9.6$ \\
\hline & $\mathrm{D}_{99}(\mathrm{~Gy})$ & $67.9 \pm 7.1$ & $56.8 \pm 7.6$ & $65.5 \pm 5.4$ & $64.2 \pm 6.3$ \\
\hline & $D_{\max }(G y)$ & $76.8 \pm 1.4$ & $71.6 \pm 4.0$ & $76.6 \pm 1.3$ & $76.6 \pm 1.3$ \\
\hline & $D_{1}(G y)$ & $76.4 \pm 1.4$ & $71.3 \pm 3.9$ & $76.3 \pm 1.3$ & $76.4 \pm 1.3$ \\
\hline \multirow[t]{6}{*}{ ITV } & V & $99.6 \pm 1.7$ & $79.8 \pm 30.4$ & $98.4 \pm 4.3$ & $97.3 \pm 5.7$ \\
\hline & $D_{\text {mean }}(G y)$ & $73.3 \pm 1.7$ & $64.8 \pm 5.2$ & $71.0 \pm 2.1$ & $70.6 \pm 2.3$ \\
\hline & $D_{\min }(G y)$ & $60.6 \pm 12.4$ & $47.7 \pm 11.0$ & $57.9 \pm 11.7$ & $56.3 \pm 11.7$ \\
\hline & $D_{99}(G y)$ & $66.0 \pm 10.5$ & $53.2 \pm 9.9$ & $62.1 \pm 8.6$ & $60.7 \pm 9.4$ \\
\hline & $D_{\max }(G y)$ & $77.0 \pm 1.5$ & $71.6 \pm 4.0$ & $76.6 \pm 1.3$ & $76.6 \pm 1.3$ \\
\hline & $D_{1}$ (Gy) & $76.7 \pm 1.4$ & $71.1 \pm 4.0$ & $76.2 \pm 1.2$ & $76.2 \pm 1.2$ \\
\hline \multirow[t]{6}{*}{ PTV } & $V_{60 G y}(\%)$ & $95.1 \pm 1.9$ & $55.7 \pm 27.0$ & $90.9 \pm 12.5$ & $85.5 \pm 15.3$ \\
\hline & $D_{\text {mean }}(G y)$ & $69.9 \pm 1.3$ & $60.0 \pm 5.7$ & $67.4 \pm 2.6$ & $66.6 \pm 3.1$ \\
\hline & $D_{\min }(G y)$ & $43.4 \pm 4.9$ & $35.8 \pm 3.9$ & $49.2 \pm 4.4$ & $46.4 \pm 5.2$ \\
\hline & $D_{99}(G y)$ & $55.0 \pm 1.9$ & $44.3 \pm 5.3$ & $56.7 \pm 3.7$ & $54.2 \pm 4.5$ \\
\hline & $D_{\max }(G y)$ & $77.0 \pm 1.5$ & $71.6 \pm 4.0$ & $76.6 \pm 1.3$ & $76.6 \pm 1.3$ \\
\hline & $D_{1}(G y)$ & $76.5 \pm 1.4$ & $70.7 \pm 4.1$ & $75.8 \pm 1.2$ & $75.8 \pm 1.2$ \\
\hline \multirow[t]{3}{*}{ Lungs } & $V_{20 \text { Gy }}(\%)$ & $7.6 \pm 4.3$ & $6.8 \pm 4.2$ & $9.6 \pm 5.0$ & $8.9 \pm 4.9$ \\
\hline & $V_{10 \text { Gy }}(\%)$ & $14.3 \pm 5.7$ & $13.4 \pm 6.3$ & $17.4 \pm 6.6$ & $16.3 \pm 6.5$ \\
\hline & $D_{\text {mean }}(G y)$ & $5.3 \pm 2.1$ & $5.0 \pm 2.0$ & $6.6 \pm 2.3$ & $6.1 \pm 2.3$ \\
\hline Heart & $D_{\text {mean }}(G y)$ & $3.0 \pm 3.5$ & $2.9 \pm 3.5$ & $3.6 \pm 3.5$ & $3.4 \pm 3.5$ \\
\hline Spinal cord & $D_{\max }(G y)$ & $8.3 \pm 8.8$ & $8.1 \pm 8.5$ & $10.9 \pm 9.6$ & $10.3 \pm 9.6$ \\
\hline Esophagus & $D_{\max }(G y)$ & $17.6 \pm 9.7$ & $16.9 \pm 8.9$ & $19.6 \pm 10.6$ & $19.3 \pm 10.3$ \\
\hline
\end{tabular}
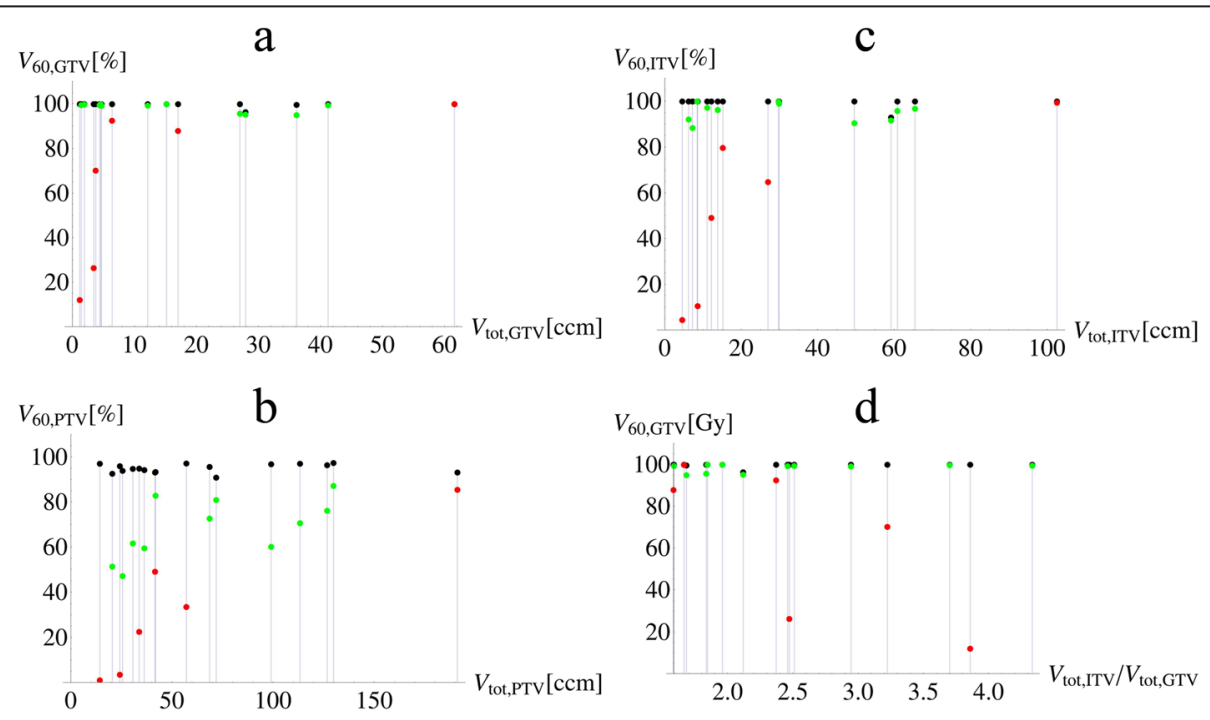

Figure 1 Target coverage versus target volume for a) gross tumor volume (GTV), b) ITV and c) planning target volume (PTV); d) shows GTV coverage versus the ratio of ITV and GTV volume which is indicative of tumor movement. The black markers indicate the original values calculated with pencil beam (PB), while the red and green markers show the recalculated collapsed cone ( $\mathrm{CC}_{\text {re-calc }}$ ) patients with central tumors (red) and tumors attached to mediastinum or pleura (green). 


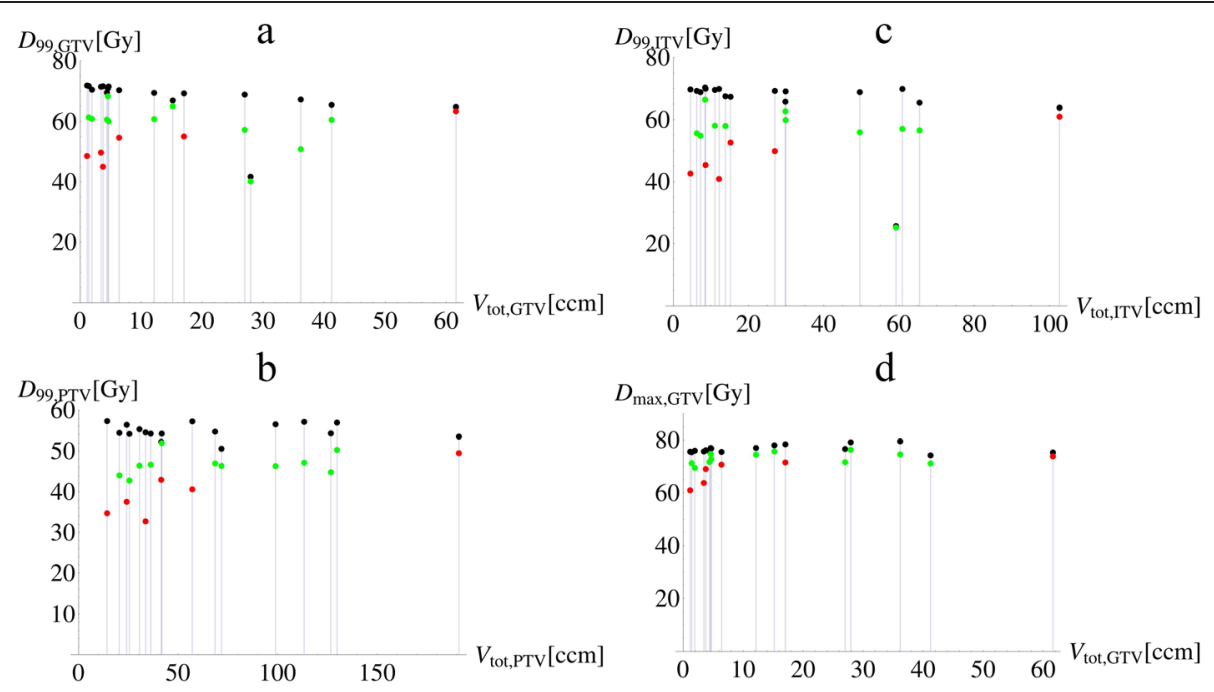

Figure $2 D_{99}$ versus the total absolute volume for a) GTV, b) ITV and c) PTV; d) shows the maximal dose versus the total GTV volume. The black markers indicate the original values calculated with pencil beam (PB), while the red and green markers show the recalculated collapsed cone $\left(\mathrm{CC}_{\mathrm{re}-\mathrm{cal}}\right)$ patients with central tumors (red) and tumors attached to mediastinum or pleura (green).

more accurately in tumors partially attached to denser tissue than in those surrounded mainly by air.

In one extreme case of a central tumor with very small volume the GTV $\mathrm{V}_{60 \mathrm{~Gy}}$ was reduced from $100 \%$ to $26 \%$, $D_{\text {mean }}$ from 74.2 Gy to 57.9 Gy and $D_{99}$ from 71.6 Gy to 49.9 Gy. The PTV coverage was tremendously reduced from $96 \%$ to $3.6 \%$. The DVHs of this patient are shown in Figure 3. Dose distributions of this patient and another less extreme case with larger tumor volume close to the posterior chest wall are shown in Figure 4.

No correlation was found between tumor movement (which was quantified by the ratio of ITV volume to GTV volume) and difference in GTV coverage (Figure 1 bottom, right, $\rho=0.12, p=0.63)$. However, this result may be limited to our study and a more rigorous analysis is necessary to evaluate the impact of tumor motion on dose distribution in general, for example by performing a 4D dose calculation study similar to Guckenberger et al. [5].

\section{PB vs. $\mathrm{CC}_{\text {clin }}$ and $\mathrm{CC}_{\text {cov }}$}

For the GTV there was no statistically significant difference in $D_{\text {mean }}, D_{\text {min }}, D_{\max }, D_{99}, D_{1}$ when comparing $P B$ to the plan with similar coverage $\left(\mathrm{CC}_{\text {cov }}\right)$. By design, there was no difference in coverage $\left(\mathrm{V}_{60 \mathrm{~Gy}}\right)$. For PTV, there was a significant difference in $\mathrm{D}_{\text {mean }}, \mathrm{D}_{\min }, \mathrm{D}_{99}$ and $D_{1}$, but not in terms of coverage and $D_{\max }$. In order to achieve similar coverage, MUs delivered and field size in $\mathrm{X}$ and $\mathrm{Y}$ direction had to be adjusted. Total MUs and average field size in $\mathrm{x}$ and $\mathrm{y}$ direction were significantly larger in the $\mathrm{CC}_{\text {clin }}$ and $\mathrm{CC}_{\text {cov }}$ plans $(\mathrm{p}<0.001$, Table 3$)$. Fields were more often adjusted craniocaudally.
In current clinical practice we use the enhanced $\mathrm{CC}$ algorithm to calculate dose, and the field size is allowed to increase up to a distance of $10 \mathrm{~mm}$ between the jaws and the outer PTV margin in BEV (compared to $5 \mathrm{~mm}$ in former plans calculated with $\mathrm{PB}$ ) to achieve more adequate calculated GTV coverage. Therefore, the clinically accepted plans $\mathrm{CC}_{\text {clin }}$ had acceptable GTV coverage that was not different from coverage calculated with the original $\mathrm{PB}$. However, $\mathrm{CC}_{\text {clin }}$ plans had decreased $\mathrm{D}_{\min }$ and $\mathrm{D}_{99}$ for the GTV.

The field size is usually not increased enough to also achieve the same planned PTV coverage as with PB, especially in areas mainly consisting of air. Thus, by construction, PTV values do not reach values of the original $\mathrm{PB}$ plan for $\mathrm{CC}_{\text {clin }}$ plans.

All evaluated parameters for the OAR were significantly larger in the $\mathrm{CC}_{\text {cov }}$ plan than $\mathrm{PB}$ and consequently in $\mathrm{CC}_{\text {re-calc. }}$. This is due to the increased monitor units and field sizes necessary to achieve similar calculated target coverage for the $\mathrm{CC}_{\text {cov }}$ plan compared to the PB plan.

The results for the $\mathrm{CC}_{\text {cov }}$ and $\mathrm{CC}_{\text {re-calc }}$ plans imply that when switching dose calculation algorithms from $\mathrm{PB}$ to $\mathrm{CC}$ without changing the criteria for an acceptable treatment plan, the OAR will be exposed to increased dose. This is because when using CC the same plan quality in terms of reported target coverage (especially in the PTV) can only be achieved by increasing MU and field size, which leads to higher dose to OAR. Although the $\mathrm{CC}$ calculated dose is closer to the truly delivered dose and thus preferable over PB, many OAR constraints originate from experience with $\mathrm{PB}$. While some of the 


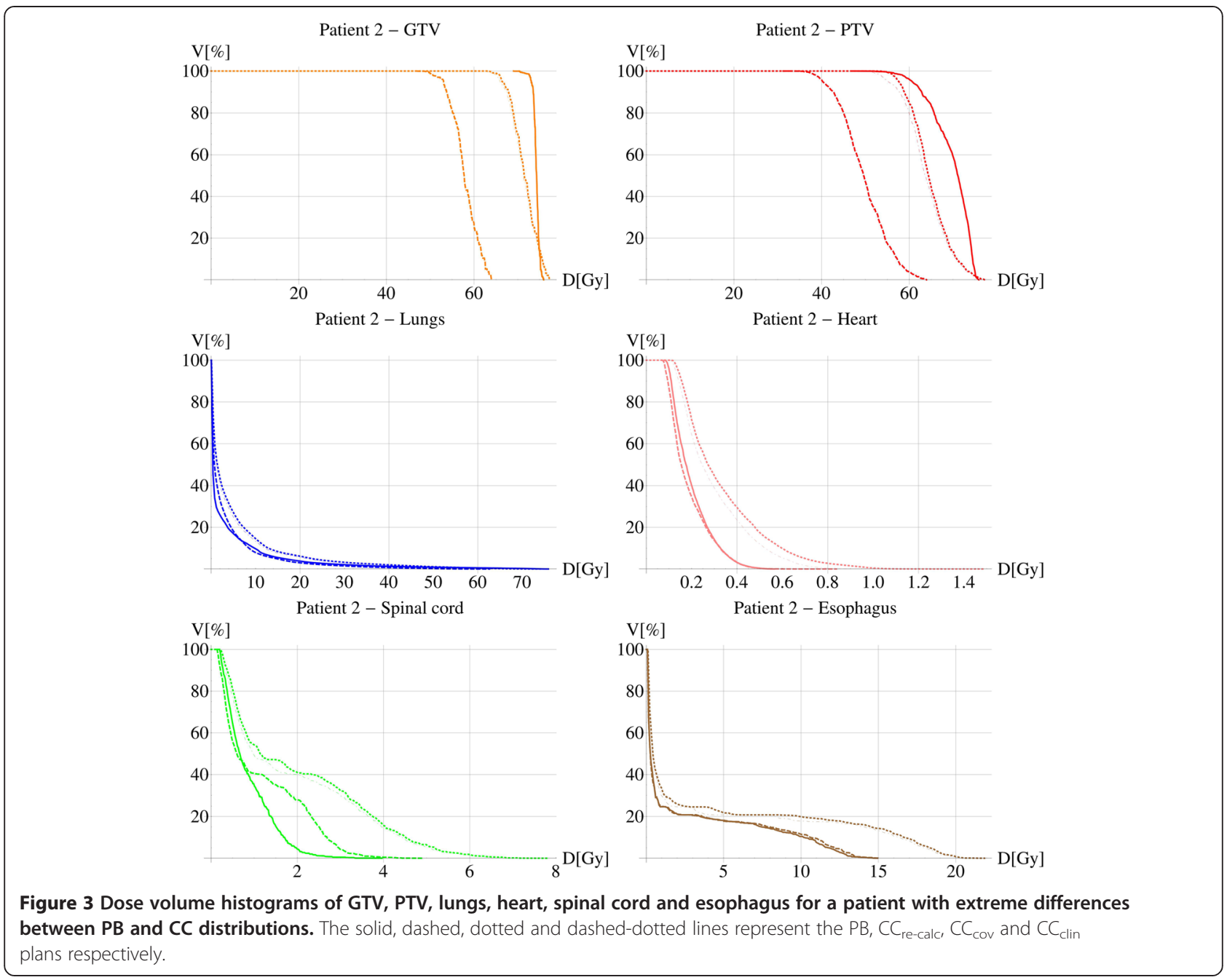

available recommendations for lung SBRT may suggest adaptation of the dose prescription according to the type of dose-calculation algorithm used that is not nearly always the case for clinical recommendations [6].

The difference between dose estimates by using $\mathrm{PB}$ and $\mathrm{CC}$ in target structures is especially pronounced in the PTV, because the PTV contains tumor tissue as well as air in the planning $\mathrm{CT}$ and thus includes large variation of tissue density. Our data is in the range of previously reported works by Haedinger et al. [7], who found a decrease in $D_{\text {mean }}$ of $11.2 \%$ whereas $14.2 \%$ was found in our study. However, we found a much more drastic change in average PTV coverage of 39.4 volume \% compared to 7.1 volume \% found by Haedinger et al., who used the Helax-TMS treatment planning system. It is therefore essential to keep stressing the importance of the choice of suitable dose calculation algorithms. Although there are a variety of studies that have investigated implications of using PB type versus CC type dose calculation algorithms (e.g. $[7,8]$ ), most publications on the matter consider the PTV and CTV mainly or exclusively. However, Guckenberger et al. [5] have shown that the 4D dose calculated over all breathing phases in the GTV is similar to the dose in the GTV in one single phase for 3D conformal plans (end-exhalation, end-inhalation, or mid-ventilation phase). This means that the GTV may really be the relevant ROI for evaluating such differences resulting from dose calculation algorithms assuming that the fields are opened enough to allow coverage of the GTV in all phases. The present study therefore also includes data for the GTV.

Aarhup and Dobler [3,4] demonstrated discrepancies in mixed-density phantom studies. They showed that PB algorithms tend to overestimate the target dose, while $\mathrm{CC}$ and MC seemed to provide more reliable data compared to measurements. Latifi et al. [9] demonstrated that there was a significantly higher rate of reoccurrence when SABR plans were planned using a PB algorithm for dose calculation than if $\mathrm{CC}$ was used.

The performance of dose calculation algorithms in lung depends on the use of different patient models [10] in order to account for target motion in the presence of 


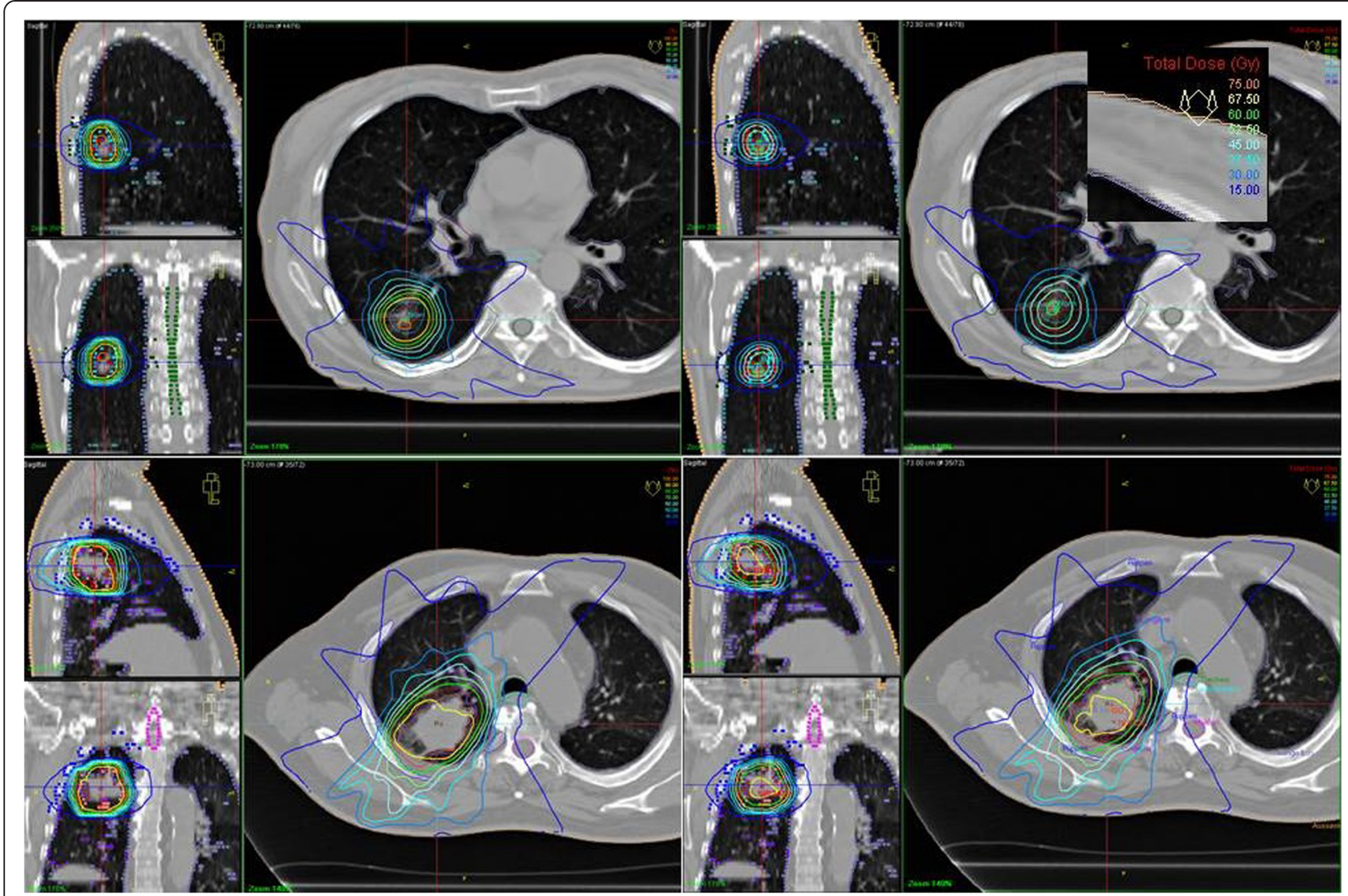

Figure 4 Sagittal, coronal and transversal CT view of two sample patients. The top slices show the most extreme case (Patient 2) due to the small tumor volume, whereas the bottom slices depict a less extreme case. Pencil beam (PB) is shown on the left and the plans recalculated with collapsed cone $\left(\mathrm{CC}_{\text {re-calc }}\right)$ on the right. The orange, yellow, bright green, light green, light blue, medium blue, turquoise and dark blue lines represent the 75 Gy, 67.5 Gy, 60 Gy, 52.5 Gy, 45 Gy, 37.5 Gy, 30 Gy and 15 Gy isodoses, respectively

large density inhomogeneities (i.e. one phase static CTs, average CTs, CTs with density overrides, maximum intensity projection) $[11,12]$. For the purpose of this study, we performed dose calculation only on a single static planning CT, which is the current clinical practice in our institution for lung SABR treatment planning using Oncentra MasterPlan ${ }^{\circ}$. Furthermore, it should be noted that both the quality of the beam model and the TPSspecific implementation of the head model can influence the performance of different dose calculation algorithms and impact the calculated dose distribution. The results obtained in this study may therefore be specifically useful for OTP users. For the purpose of our study we used PB- and CC-beam models that were carefully verified

Table 3 Relative change in average total monitor units with respect to $\mathrm{PB}$ plans and average field size

\begin{tabular}{llll}
\hline & $\mathbf{P B}$ & $\mathbf{C C}_{\text {cov }}$ & $\mathbf{C C}_{\text {clin }}$ \\
\hline Average relative change of total MU (\%) & - & $5.0 \pm 5.6$ & $5.8 \pm 5.9$ \\
Average field size X $(\mathrm{cm})$ & $4.6 \pm 1.3$ & $5.0 \pm 1.3$ & $4.9 \pm 1.3$ \\
Average field size $\mathrm{Y}(\mathrm{cm})$ & $6.5 \pm 1.9$ & $7.2 \pm 1.9$ & $6.9 \pm 2.0$ \\
\hline
\end{tabular}

with respect to base data measurements prior to clinical use in our institution.

The large discrepancies resulting from use of different dose calculation algorithms are of special importance when multi-institutional studies are performed. A comparative planning study for the JCOG 0403 protocol showed notable differences between the participating institutions for $D_{\max }, D_{\min }, D_{95}$, and the homogeneity index of the PTV, although target definitions and target dose constraints were the same. These inter-institutional deviations were mainly attributed to the different choice of dose calculation algorithms used in the institutions [13]. Even if only CC algorithms are used, their quality may depend on the exact implementation of the algorithm in the treatment planning system, and be specific to the release version $[14,15]$. The use of a wide variety of dose prescription modes in stereotactic radiotherapy leads to additional intransparency when comparing data [16].

More recently started studies require tissue density heterogeneity correction. For RTOG 0236, SBRT conformal treatment plans were generated using $\mathrm{XiO} /$ superposition meeting dosimetric compliance criteria recommended for 
RTOG 0813 and recalculated using MC. Tissue density heterogeneity correction was applied in the initial calculations. V20Gy increased on average by $18 \%$ in the MC plans [17]. Although CC algorithms predict dose more accurately than $\mathrm{PB}$ algorithms, they can nevertheless deviate from measured dose and dose calculated with MC. Several studies investigated deviations of CC algorithms from measured dose or dose calculated with MC (e.g. [2,15,18]). Krieger et al. [2] and Kry et al. [18] found good agreement between MC and measured dose. Thus, in order to fully evaluate the implications of using $\mathrm{PB}$ and $\mathrm{CC}$ algorithms in the clinic, a comparison with MC would be necessary.

\section{Conclusions}

The use of different calculation algorithms leads to significant changes not only in dose, but also in field size and MUs delivered, if similar target coverage criteria are applied for treatment planning. This has to be taken into account for treatment planning and comparison of data concerning side effects as well as local control in radiotherapy of intrapulmonary lesions. Using CC improves the accuracy of dose calculation in the tumor. However, attempting to cover the parts of the PTV and ITV that mainly consist of air, caused a higher strain on the lung and OARs in our study. In cases with borderline acceptable OAR exposition, CC plans should be evaluated cautiously.

If data concerning safety margins, OAR limits, maximum or mean dose and PTV/ITV coverage of radiotherapy plans in stereotactic radiotherapy are compared, it is of interest to state which dose calculation algorithm was used. Future projects will include comparison of the resulting $\mathrm{CC}$ and $\mathrm{PB}$ dose distributions to the $\mathrm{MC}$ gold standard, as dose calculated with CC itself can deviate from measured values. Also evaluation of accumulated 4D dose may be necessary to estimate the actual increase of TCP that can be achieved by trying to obtain PTV coverage with $\mathrm{CC}$.

\section{Abbreviations}

CC: Collapsed cone; PB: Pencil beam; MC: Monte Carlo; OAR: Organs at risk; SABR: Stereotactic ablative radiotherapy; DVH: Dose volume histogram; PTV: Planning target volume; IT: Internal target volume; CTV: Clinical target volume; GTV: Gross tumor volume; MU: Monitor unit; ROI: Region of interest; MLC: Multi leaf collimator; TCP: Tumor control probability; BEV: Beam's eye view; TPS: Treatment planning system.
\end{abstract}

\section{Competing interests}

The authors declare that they have no competing interests.

\section{Authors' contributions}

SP, AT, SGa, SGe, MS, UG and CB planned, coordinated and performed the study. AT, SGa, SP and SK collected and analyzed the data. AT and SP prepared the manuscript. MS and SK revised the manuscript. All authors have read and approved the final manuscript.

Received: 7 August 2014 Accepted: 9 February 2015

Published online: 22 February 2015

\section{References}

1. Ahnesjö A, Aspradakis MM. Dose calculations for external photon beams in radiotherapy. Phys Med Biol. 1999;44:R99-155.

2. Krieger T, Sauer OA. Monte Carlo- versus pencil-beam-/collapsed-cone-dose calculation in a heterogeneous multi-layer phantom. Phys Med Biol. 2005;50:859-68.

3. Aarup LR, Nahum AE, Zacharatou C, Juhler-Nøttrup T, Knöös T, Nyström H, et al. The effect of different lung densities on the accuracy of various radiotherapy dose calculation methods: implications for tumour coverage. Radiother Oncol J Eur Soc Ther Radiol Oncol. 2009:91:405-14.

4. Dobler B, Walter C, Knopf A, Fabri D, Loeschel R, Polednik M, et al. Optimization of extracranial stereotactic radiation therapy of small lung lesions using accurate dose calculation algorithms. Radiat Oncol Lond Engl. 2006;1:45.

5. Guckenberger M, Wilbert J, Krieger T, Richter A, Baier K, Meyer J, et al. Four-dimensional treatment planning for stereotactic body radiotherapy. Int J Radiat Oncol Biol Phys. 2007:69:276-85.

6. Hurkmans CW, Cuijpers JP, Lagerwaard FJ, Widder J, van der Heide UA, Schuring $D$, et al. Recommendations for implementing stereotactic radiotherapy in peripheral stage IA non-small cell lung cancer: report from the Quality Assurance Working Party of the randomised phase III ROSEL study. Radiat Oncol Lond Engl. 2009;4:1.

7. Haedinger $U$, Krieger T, Flentje M, Wulf J. Influence of calculation model on dose distribution in stereotactic radiotherapy for pulmonary targets. Int J Radiat Oncol Biol Phys. 2005;61:239-49.

8. Traberg Hansen A, Petersen JB, Høyer M, Christensen JJ. Comparison of two dose calculation methods applied to extracranial stereotactic radiotherapy treatment planning. Radiother Oncol J Eur Soc Ther Radiol Oncol. 2005;77:96-8.

9. Latifi K, Oliver J, Baker R, Dilling TJ, Stevens CW, Kim J, et al. Study of 201 non-small cell lung cancer patients given stereotactic ablative radiation therapy shows local control dependence on dose calculation algorithm. Int J Radiat Oncol. 2014;88:1108-13.

10. Sikora M, Muzik J, Söhn M, Weinmann M, Alber M. Monte Carlo vs Pencil Beam based optimization of stereotactic lung IMRT. Radiat Oncol. 2009;4:64.

11. Bradley JD, Nofal AN, El Naqa IM, Lu W, Liu J, Hubenschmidt J, et al. Comparison of helical, maximum intensity projection (MIP), and averaged intensity (AI) 4D CT imaging for stereotactic body radiation therapy (SBRT) planning in lung cancer. Radiother Oncol. 2006;81:264-8.

12. Pan T, Riegel AC, Ahmad MU, Sun X, Chang JY, Luo D. New weighted maximum-intensity-projection images from cine $\mathrm{CT}$ for delineation of the lung tumor plus motion. Med Phys. 2013;40:061901.

13. Matsuo Y, Takayama K, Nagata Y, Kunieda E, Tateoka K, Ishizuka N, et al. Interinstitutional variations in planning for stereotactic body radiation therapy for lung cancer. Int J Radiat Oncol Biol Phys. 2007;68:416-25.

14. Huang JY, Eklund D, Childress NL, Howell RM, Mirkovic D, Followill DS, et al. Investigation of various energy deposition kernel refinements for the convolution/superposition method. Med Phys. 2013;40:121721.

15. Vanderstraeten B, Reynaert N, Paelinck L, Madani I, De Wagter C, De Gersem W, et al. Accuracy of patient dose calculation for lung IMRT: A comparison of Monte Carlo, convolution/superposition, and pencil beam computations. Med Phys. 2006;33:3149-58.

16. Onishi H, Shirato H, Nagata Y, Hiraoka M, Fujino M, Gomi K, et al. Hypofractionated stereotactic radiotherapy (HypoFXSRT) for stage I non-small cell lung cancer: updated results of 257 patients in a Japanese multi-institutional study. J Thorac Oncol. 2007;2:S94-100.

17. Li J, Galvin J, Harrison A, Timmerman R, Yu Y, Xiao Y. Dosimetric verification using monte carlo calculations for tissue heterogeneity-corrected conformal treatment plans following RTOG 0813 dosimetric criteria for lung cancer stereotactic body radiotherapy. Int J Radiat Oncol Biol Phys. 2012;84:508-13.

18. Kry SF, Alvarez P, Molineu A, Amador C, Galvin J, Followill DS. Algorithms used in heterogeneous dose calculations show systematic differences as measured with the Radiological Physics Center's anthropomorphic thorax phantom used for RTOG credentialing. Int J Radiat Oncol. 2013;85:e95-100. 